\title{
BMJ Open Hospital climate actions and assessment tools: a scoping review protocol
}

\author{
Connie Cai Ru Gan (D , , Nicola Banwell, ${ }^{2}$ Ramon San Pascual, ${ }^{3}$ Cordia Chu, ${ }^{1}$ \\ Ying Wei Wang ${ }^{4}$
}

To cite: Gan CCR, Banwell N, Pascual RS, et al. Hospital climate actions and assessment tools: a scoping review protocol. BMJ Open 2019;9:e032561. doi:10.1136/ bmjopen-2019-032561

- Prepublication history for this paper is available online. To view these files, please visit the journal online (http://dx.doi. org/10.1136/bmjopen-2019032561).

Received 25 June 2019 Revised 27 November 2019 Accepted 05 December 2019

Check for updates

C Author(s) (or their employer(s)) 2019. Re-use permitted under CC BY-NC. No commercial re-use. See rights and permissions. Published by BMJ.

${ }^{1}$ Centre for Environment and Population Health, Griffith University, Nathan, Queensland, Australia

${ }^{2}$ Cooperation and Development, École Polytechnique Fédérale de Lausanne, Lausanne, VD, Switzerland

${ }^{3}$ Health Care Without Harm, Manila, Philippines

${ }^{4}$ Health Promotion

Administration Ministry of Health and Welfare, Taipei City, Taiwan

Correspondence to Professor Ying Wei Wang; drywwang@gmail.com

\begin{abstract}
Introduction Health and climate change are inexorably linked through the exacerbation of health risks and the contribution of the health sector to greenhouse gas emissions. Climate action in healthcare settings is critical to reduce risks and impacts of climate change through the smarter use of energy, minimising waste and enhancing disaster preparedness. Globally, hospital climate action is growing; however, the potential for further progress and impacts remains. The literature on this topic lacks synthesis, and this poses challenges for hospital leadership in tracking the impact of climate action. This scoping review will summarise the current knowledge about hospital climate action and existing tools to measure progress in this area.
\end{abstract}

Methods and analysis This scoping review will be conducted applying the six-stage protocol proposed by Arksey and 0'Malley. The study includes literature of how hospitals have addressed climate change (mitigation and adaptation) since the Kyoto Protocol was signed in 1997. All identified studies indexed in Medline, Scopus, Embase and CINAHL will be examined. The search strategy will also include Google Scholar to capture relevant grey literature. Quantitative and thematic analysis will be used to evaluate and categorise the study results.

Ethics and dissemination This scoping review is part of the climate-smart healthcare initiative which will provide a valuable synthesis to aid understanding of hospitals' climate actions, and tools used to measure its implementation. As such it will contribute to mobilising and accelerating the implementation of climate action in hospitals. The findings will be disseminated with all members of the International Health Promoting Hospital and Health Services (HPH) and the Global Green and Healthy Hospital network. Dissemination will occur through peer-reviewed publications; and with the HPH and GGHH members through its annual conference and newsletter.

\section{INTRODUCTION}

Climate change threatens the lives and health of countless people, coastal cities and local economies. Researchers have warned that climate change will intensify and increase the severity of various climatesensitive extreme events and their associated health risks. ${ }^{1-4}$ More than 6000 subnational actors, including cities and regions, have made quantifiable commitments to reducing emissions to achieve the Paris Agreement. ${ }^{5}$
Strengths and limitations of this study

This systematic scoping review will provide a pertinent synthesis and systematic examination of scientific and grey literature regarding mitigation and adaptation in hospitals.

- This review will study multidisciplinary databases covering public health, medicine, engineering, architecture, social science, policy and the environment to provide a comprehensive evaluation of the literature.

- There will be no restrictions applied to healthcare facility type, study design or location which are published in English, Chinese and Indonesian.

- The study aims to synthesise different aspects of hospital climate actions; identified studies will not be excluded based on quality assessment. However, the type of literature and the value of available evidence will be charted in the review.

- While there is limited to no incentive for hospital to publish actions taken on adaptation and mitigation, data sources are restricted only to those that were publicly available. However, relevant stakeholders' insights will be incorporated into this review process.

However, making commitments is only the first step towards the transformations necessary to achieve nationally determined contributions. Collective tracking of contributions from individual commitments is challenging because of inadequate and often incomplete information on targets and greenhouse gas emissions. Moreover, there are ongoing efforts to align methodologies and concepts applied by the diverse range of stakeholders engaged in climate action.

Delays in action increase the risk of missing global mitigation targets and exposing the world to more significant impacts from climate change. ${ }^{6}$ The recent Intergovernmental Panel on Climate Change '1.5 Degree Report' highlighted the potential consequences of climate change if current emission rates continue. ${ }^{8}$ Global warming and climaterelated disasters, such as floods and infectious disease outbreaks present severe health threats on a global scale. ${ }^{9}$ Hospitals play a 
Box 1 Search terms for the scoping review on 'hospital and climate action'

\section{Hospital-related}

- Healthcare provider/industry/sector, hospital, medical facility/institution/centre/acute care unit, intensive care unit, ward, clinic, infirmary, emergency department, trauma centre, nursing station, operating theatre, dialysis centre, operating room, surgical services.

\section{Climate-related}

- Climate change, global warming, extreme weather, climate variability, greenhouse gas emission, greenhouse effect, high temperature, heatwave, drought, flood, climate-induced, climate-related disaster, storm, typhoon, hurricanes, cyclone, sea-level rise

\section{Climate Action-related}

- Mitigation, reduction, adaptation, cease, tackle, address, combat, fight, strategy, procedure, process, implementation, action, effort, attempt, policy, framework, plan, law, approach, response, system thinking, integrated model.

critical role in reducing health impacts by (1) treating illnesses and injuries, (2) being prepared for climateinduced disasters, (3) effectively engage the community on adaptation activities and (4) stepping up to minimise healthcare carbon emissions. ${ }^{10} 11$

Healthcare is among the 'heavy-emitting' sectors; the global healthcare sector had a climate footprint of 2.0 gigatonnes of equivalent of carbon dioxide $\left(\mathrm{GtCO}_{2} \mathrm{e}\right)$ in 2014 , equivalent to $4.4 \%$ of global net emissions. ${ }^{1 \mathrm{e}}$ The National Health Service carbon footprint in England is 22.8 million tonnes of carbon dioxide equivalents $\left(\mathrm{MtCO}_{2} \mathrm{e}\right)$ in 2015 . $^{12}$ The Australian healthcare sector accounts for $7 \%$ of total national emissions ${ }^{13}$ and $10 \%$ of the United States ${ }^{14}$ national $\mathrm{CO}_{2}$ e emissions. As hospitals are often significant consumers of energy and producers of waste, ${ }^{15}$ several organisations and alliances in North America, Europe and Asia have surfaced over the past decade with commitments addressing these issues. ${ }^{16-19}$

Hospitals must be encouraged to uphold their mitigation efforts, and there are mitigation measures which hospitals can take to slow the warming trend. Lowcarbon health services including hospital design which allow natural lighting, policies that prioritise renewable energy transition, employs energy efficiency measures, ${ }^{20}$ rainwater harvesting, provision of facilities for cycling or walking and minimise healthcare waste management ${ }^{21}$ and refusing single-use plastics with green procurement policy. ${ }^{22}$ The healthcare sector cannot turn away from climate change and must act on this responsibility through accelerated concrete implementation of carbon reduction initiatives that also improve the standard of healthcare. ${ }^{23}$ However, at this critical time, mitigating emissions is no longer optional; furthermore, specific adaptation actions are necessary to face climate-health threats.

The healthcare sector has the responsibility to act on health threats and manage future demands on the health service. Healthcare facilities need to incorporate both mitigation and adaptation measures, which include projections and preparedness for the impacts of climate change to lessen health burden. By focusing their efforts to enhance the adaptive capacity in specific areas, promoting institutional improvement, embracing adaptive management and developing tools ${ }^{24}$ to support these processes are important priorities and can build the resilience of local health systems to climate change. ${ }^{2526}$ However, it is unclear how hospitals worldwide are acting to reduce emissions and adapt to climate change.

As the converging crises of health and climate change, we require much more radical climate action. Therefore, this scoping review will synthesise the current knowledge about hospital climate action and the existing tools to measure progress in this area. As such, it will contribute to mobilising and accelerating hospitals to implement climate action.

\section{RESEARCH OBJECTIVES}

The purpose of this scoping review is to synthesise knowledge on how hospitals incorporate adaptation and mitigation practices to address climate change. The findings from this scoping review will provide a comprehensive understanding of the current knowledge of hospital climate actions and tools used to measure their implementation. This review will showcase existing actions and share knowledge on solutions with high potential for success.

\section{METHODOLOGY}

\section{Patient and public involvement}

This scoping review study will have no patient or public involvement in the design or planning of this study.

\section{Protocol design}

This scoping review will be conducted based on the methodology as per Arksey and O'Malley ${ }^{27}$ and further developed by Levac et al..$^{28}$ These methods include sixstage procedures including (1) identifying the research question; (2) information sources and search strategy; (3) study selection; (4) data extraction; (5) collating, summarising and reporting the result; and (6) external consultation with relevant stakeholders.

1. Identifying the research question

- To what extent do hospitals address climate change?

- Mitigation (sustainable policy, improve energy efficiency, shifting to renewable energy, minimising waste, green procurement).

- Adaptation (disaster risk reduction, health professionals' and community climate awareness and preparedness, vulnerability evaluation, early warning system).

- What measures and tools exist in the scientific literature regarding climate change mitigation and adaptation in hospitals?

2. Information sources and search strategy 
Table 1 Search strategy syntax for Medline, Scopus, Embase and CINAKL

\section{Database Search strategy syntax}

\begin{tabular}{|c|c|}
\hline Medline & 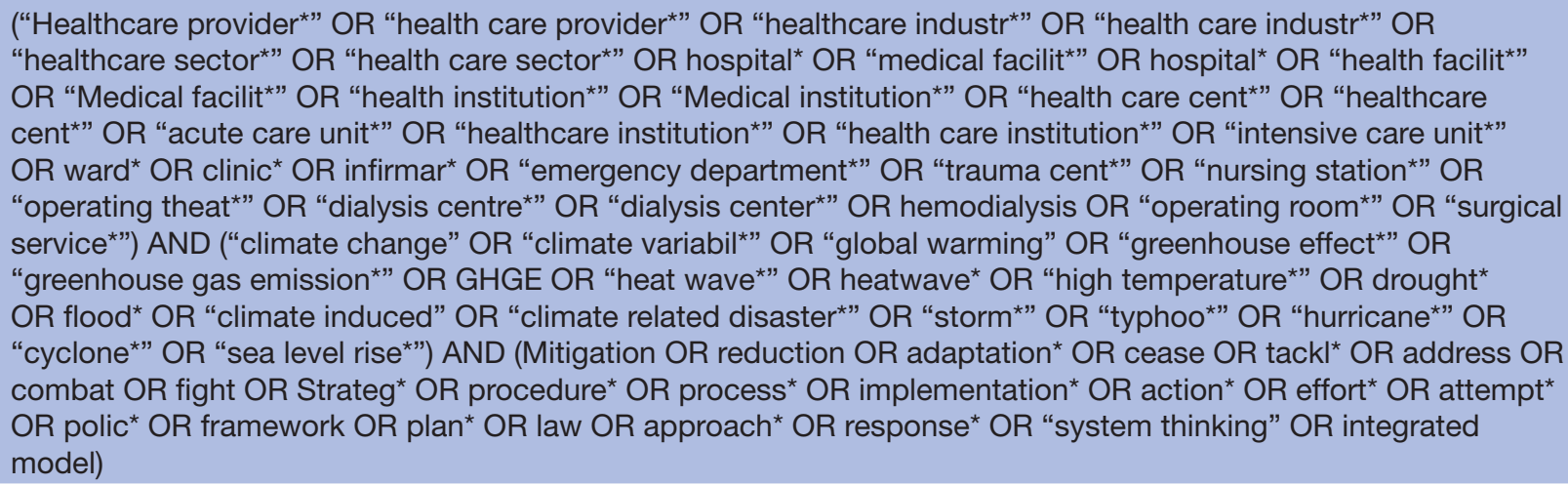 \\
\hline
\end{tabular}

Scopus TITLE-ABS-KEY ("Healthcare provider" OR "health care provider" OR "healthcare industr" OR "health care industr" OR "healthcare sector" "OR "health care sector"” OR hospital* OR "medical facilit" OR hospital" OR "health facilit" OR "Medical facilit" OR "health institution"” OR "Medical institution" OR "health care cent" OR "healthcare cent" ${ }^{\star}$ OR "acute care unit" OR "healthcare institution*" OR "health care institution" OR "intensive care unit*" OR ward ( OR clinic $^{\star}$ OR infirmar ${ }^{\star}$ OR "emergency department*" OR "trauma cent*" OR "nursing station" OR "operating theat" OR "dialysis centre*” OR "dialysis center" OR hemodialysis OR "“"operating room"” OR "surgical service*") AND TITLE-ABS-KEY ("climate change" OR "climate variabil" OR "global warming" OR "greenhouse effect*" OR "greenhouse gas emission"” OR ghge OR "heat wave*" OR heatwave* OR "high temperature*" OR drought* OR flood* OR "climate induced" OR "climate related disaster*" OR storm* OR typhoo* OR hurricane* OR cyclone* OR sea level rise*) AND TITLE-ABS-KEY (mitigation OR reduction OR adaptation* OR cease OR tackl ${ }^{\star}$ OR address OR combat OR fight OR strateg* OR procedure* OR process ${ }^{\star}$ OR implementation* OR action* OR effort* OR attempt* OR polic* OR framework OR plan* OR law OR approach* OR response* OR "system thinking" OR integrated model*)

Embase ('healthcare provider':ti,ab,kw OR 'health care provider':ti,ab,kw OR 'healthcare industr':ti,ab,kw OR 'health care industr':ti,ab,kw OR 'healthcare sector" ${ }^{\star *}$ ti,ab,kw OR 'health care sector ${ }^{\star 1}: t i, a b, k w$ OR hospital*:ti,ab,kw OR 'health facilit' :ti,ab,kw OR 'medical facilit':ti,ab,kw OR 'health institution*':ti,ab,kw OR 'medical institution ${ }^{\star 1}: t i, a b, k w$ OR 'health care cent ${ }^{\star 1}: t i, a b, k w$ OR 'healthcare cent ${ }^{\star \prime}: t i, a b, k w$ OR 'acute care unit' $: t i, a b, k w$ OR 'healthcare institution*':ti,ab,kw OR 'health care institution*1:ti,ab,kw OR 'intensive care unit':ti,ab,kw OR ward $^{*}: t i, a b, k w$ OR clinic*:ti,ab,kw OR infirmar*:ti,ab,kw OR 'emergency department*':ti,ab,kw OR 'trauma

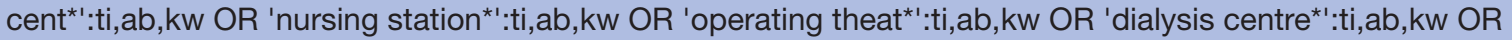
hemodialysis:ti,ab,kw) AND ('climate change':ti,ab,kw OR 'climate variabil*':ti,ab,kw OR 'global warming':ti,ab,kw OR 'greenhouse effect ${ }^{\star \prime}: t i, a b, k w$ OR 'greenhouse gas emission*':ti,ab,kw OR ghge:ti,ab,kw OR 'heat wave*':ti,ab,kw OR 'heatwave*':ti,ab,kw OR 'high temperature*':ti,ab,kw OR drought*:ti,ab,kw OR flood*:ti,ab,kw OR 'climate induced':ti,ab,kw OR 'climate related disaster”':ti,ab,kw OR storm*:ti,ab,kw OR typhoo*:ti,ab,kw OR hurricane*:ti,ab,kw OR cyclone*:ti,ab,kw OR sea level rise*:ti,ab,kw) AND (mitigation:ti,ab,kw OR reduction:ti,ab,kw OR adaptation*:ti,ab,kw OR cease:ti,ab,kw OR tackı*:ti,ab,kw OR address:ti,ab,kw OR combat:ti,ab,kw OR fight:ti,ab,kw OR strateg*:ti,ab,kw OR procedure*:ti,ab,kw OR process*:ti,ab,kw OR implementation*:ti,ab,kw OR action*:ti,ab,kw OR effort*:ti,ab,kw OR attempt*:ti,ab,kw OR polic*:ti,ab,kw OR framework:ti,ab,kw OR plan*:ti,ab,kw OR law:ti,ab,kw OR approach*:ti,ab,kw OR response*:ti,ab,kw OR system thinking*:ti,ab,kw OR integrated model $^{*}$ ti, ab,kw)

CINAKL ("Healthcare provider" OR "health care provider" OR "healthcare industr" OR "health care industr" OR "healthcare sector" OR "health care sector" OR hospital* OR "medical facilit" OR hospital* OR "health facilit" OR "Medical facilit" OR "health institution"” OR "Medical institution"” OR "health care cent" OR "healthcare cent" " OR "acute care unit*" OR "healthcare institution"” OR "health care institution" OR "intensive care unit" OR ward* OR clinic* OR infirmar* OR "emergency department" OR "trauma cent ${ }^{\star}$ " OR "nursing station" OR "operating theat"” OR "dialysis centre*" OR "dialysis center" OR hemodialysis OR "operating room" OR "surgical service") AND ("climate change" OR "climate variabil"” OR "global warming" OR "greenhouse effect" OR "greenhouse gas emission"” OR GHGE OR "heat wave" OR heatwave* OR "high temperature " OR drought* OR flood" OR "climate induced" OR "climate related disaster" OR "storm"” OR "typhoo" OR "hurricane*" OR "cyclone*" OR "sea level rise*") AND (Mitigation OR reduction OR adaptation* OR cease OR tackl* OR address OR combat OR fight OR Strateg* OR procedure* OR process* OR implementation* OR action* OR effort* OR attempt* OR polic* OR framework OR plan* OR law OR approach* OR response* OR "system thinking" OR integrated model $\left.^{*}\right)$ 
Table 2 Selection of articles based on inclusion and exclusion criteria

Inclusion criteria

Peer-reviewed articles with all types of methods

Exclusion criteria

conference proceedings, dissertations

Publication types (original research article; reviews; commentaries; editorials; case The language used does not include studies; and grey literature including conference papers, corporate sustainability English, Chinese or Indonesian. reports, theses and technical guidance documents)

Indexed in MEDLINE, Scopus and Embase

Published since 1997*

No restriction to geographical origin or population

*Date of publication: The Kyoto Protocol, an international agreement signed in 1997 (UN Doc FCCC/CP/1997/7/Add.1, Dec. 10, 1997; 37 ILM 22 (1998) which linked to the United Nations Framework Convention on Climate Change (UNFCCC), which commits its Parties by setting internationally binding emission reduction targets.

Eligible studies will be identified through Medline, Scopus, Embase and CINAHL. The search terms cover all areas of climate action and hospital, including Medical Subject Headings (MeSH) terms, subject headings and keywords. To truly capture grey literature, search strategy will include Google Scholar, and searches of targeted organisations, file types and websites. The keywords for the search strategy are outlined in box 1, and the corresponding search syntaxes for each database are outlined in table 1. To capture all eligible studies, we will follow the search strategy, which allows both MeSH terms and free text.

3. Study selection

The research team will first use Zotero software to remove duplicates of references; and then screen titles and abstracts in English, Chinese and Indonesian. Two independent reviewers who have proficiencies in English, Chinese and Indonesian will be involved in this step. Title and abstract screening will be based on the inclusion and exclusion criteria outlined in table 2. Those that meet all eligibility criteria will be included in the analysis. All screening and data extraction process will be conducted by two independent reviewers. The two primary reviewers will resolve any inconsistencies in study eligibility through discussion, before involving a third reviewer in the study selection stage.

4. Data extraction process

The scoping review will explore the implemented mitigation and adaptation actions in hospitals and the tools which are being used to track progress. Data extraction will include: author, publication date, journal, study characteristic, intervention characteristic, tools used to measure intervention, intervention results (eg, barriers, success factors and outcomes) and drivers reported associated with hospital climate action. Examples of facilitating factors include, but are not limited to, law and policy, available funding, electricity cost, advocates (eg, environmentalist), characteristic of the hospital (eg, faithbased organisations), vulnerability to climate change (eg, higher risk of flood, past disasters experience). The research team will discuss these factors before reviewing to ensure standardisation and comprehensiveness.
5. Collating, summarising and reporting the result

The primary researcher will conduct a thematic analysis to explore and examine study results, disciplines associated with hospitals climate action and tools used to measure its implementation. The result is presented using tabulated data, and new themes will be included as required.

6. External consultation with relevant stakeholders

A 1-day consultation meeting will be held to present a preliminary summary of the state of evidence related to hospital climate actions and tools used to measure its implementation. Experts, health practitioners and relevant stakeholders in the field of greening healthcare will be encouraged to express their ideas as they pertain to the results during the meeting discussion. The meeting will include a combination of structured presentations from experts, small and large group discussion sessions to facilitate knowledge exchange of insights for establishing a common consensus and identifying critical considerations for promoting climate actions in hospital settings.

The authors will use the Preferred Reporting Items for Systematic Reviews and Meta-Analyses extension for scoping review checklist ${ }^{29}$ on the reporting of this scoping review.

\section{Ethics and dissemination}

This project is literature-based research, thus does not require ethical approval. This research is part of the climate-smart hospital initiative to enhance hospital climate preparedness. The Task Force on Health Promoting Hospital and Health Services (HPH) and Environment of The International HPH provides valuable guidance to inform this work. The taskforce consists of health practitioners across regions who provide strategic advice to HPH members on various environment-related matters that impact health promotion-based practice in hospital settings.

We will share the review findings with all members of the International HPH and Global Green and Healthy Hospital network. Results may be of interest to Ministries of Health, policy-makers, national climate change and health teams, hospital managers, healthcare practitioners 
globally who are seeking to improve their understanding of hospital adaptation and implementation strategy internationally. Dissemination will occur through peerreviewed scientific publications; and with $\mathrm{HPH}$ and GGHH members through their annual conference and newsletter.

\section{Twitter Connie Cai Ru Gan @connie_gan}

Acknowledgements The authors are grateful to Wendy Summers-Penny, Dr Sima Barmania and Dr Febi Dwirahmadi for their inputs, and we thank the four reviewers whose insightful comments have greatly improved this protocol.

Contributors CCRG prepared the first draft, initiated the project and revised the manuscript. NB and RSP advised on the rationale and search strategy. CC and YWW provided inputs on study methodology.

Funding The authors have not declared a specific grant for this research from any funding agency in the public, commercial or not-for-profit sectors.

Competing interests None declared.

Patient consent for publication Not required.

Provenance and peer review Not commissioned; externally peer reviewed.

Open access This is an open access article distributed in accordance with the Creative Commons Attribution Non Commercial (CC BY-NC 4.0) license, which permits others to distribute, remix, adapt, build upon this work non-commercially, and license their derivative works on different terms, provided the original work is properly cited, appropriate credit is given, any changes made indicated, and the use is non-commercial. See: http://creativecommons.org/licenses/by-nc/4.0/.

\section{ORCID iD}

Connie Cai Ru Gan http://orcid.org/0000-0002-2252-349X

\section{REFERENCES}

1 Curtis S, Fair A, Wistow J, et al. Impact of extreme weather events and climate change for health and social care systems. Environ Health 2017;16:23-32.

2 de' DF, Michelozzi P, Change C. Climate Change, Extreme Weather Events and Health Effects. In: Goffredo S, Dubinsky Z, eds. The Mediterranean sea. Netherlands: Springer, 2014: 617-24. http://link. springer.com/chapter/

3 Qin J, Zhang J. The impacts of extreme events of weather and climate on infectious disease. J Hyg Res 2009;38:762-4.

4 Solomon CG, LaRocque RC. Climate change - a health emergency. N Engl J Med 2019;380:209-11.

5 Kuramochi T, Lui S, Höhne N, et al. Global climate action from cities, regions and businesses: impact of individual actors and cooperative initiatives on global and national emissions. 2019 edition. NewClimate Institute, data-driven lab, PBL, German development Institute/Deutsches Institut für Entwicklungspolitik (die), Blavatnik school of government, University of Oxford, 2019. Available: https:// newclimate.org/2019/09/18/global-climate-action-from-citiesregions-and-businesses-2019/

6 Beggs PJ, Zhang Y. The MJA-Lancet Countdown on health and climate change: Australian policy inaction threatens lives(Summary). Med J Aust 2018;209:474-5.
7 Watts N, Amann M, Ayeb-Karlsson S, et al. The Lancet countdown on health and climate change: from 25 years of inaction to a global transformation for public health. The Lancet 2017;0.

8 Ebi K, Campbell-Lendrum D, Wyns A. The1.5 Health Report: Synthesis on Health \& Climate Science in the IPCC SR1.5, 2018. Available: https://www.who.int/globalchange/181008_the_1_5_ healthreport.pdf

9 Louis VR, Phalkey RK. Health impacts in a changing climate - an overview. Eur Phys J Spec Top 2016;225:429-41.

10 Salas RN, Solomon CG. The Climate Crisis - Health and Care Delivery. N Engl J Med 2019;381.

11 Karliner J, Slotterback S, Boyd R, et al. Health Care's Climate Footprint. Health Care Without Harm and ARUP 2019.

12 Sustainable Development Unit. Carbon footprint update for NHS in England 2016;2015.

13 Malik A, Lenzen M, McAlister S, et al. The carbon footprint of Australian health care. Lancet Planet Health 2018;2:e27-35.

14 Frumkin H. The US Health Care Sector's Carbon Footprint: Stomping or Treading Lightly? Am J Public Health 2018;108:S56-7.

15 Chauhan A, Singh A. Healthcare waste management: a state-of-the-art literature review. Int J Environ Waste Manag 2016;18:120-44.

16 Dhillon VS, Kaur D, Hospital G. And climate change: their interrelationship and the way forward. J Clin Diagn Res JCDR 2015;9:LE01-5.

17 Gerwig K. Greening Health Care: How Hospitals Can Heal the Planet. Oxford University Press, 2014.

18 Williams A, MacNaughton P, et al. Building evidence for health: green buildings, current science, and future challenges. Annu Rev Public Health.

19 Primozic L. Greening Australia's public health system: the role of public hospitals in responding to climate change. J Law Med 2010;17:772-83.

20 P. M, X. C, J. B, et al. Energy savings, emission reductions, and health co-benefits of the green building movement. $J$ Expo Sci Environ Epidemiol 2018;28:307-18.

21 Lee B-K, Ellenbecker MJ, Moure-Eraso R. Analyses of the recycling potential of medical plastic wastes. Waste Manag 2002;22:461-70.

22 Paraschiv M, Kuncser R, Tazerout M, et al. New energy value chain through pyrolysis of hospital plastic waste. Appl Therm Eng 2015;87:424-33.

23 FitzGerald GJ, Capon A, Aitken P. Resilient health systems: preparing for climate disasters and other emergencies. Med $\mathrm{J}$ Aust 2019;210:304-5.

24 Paterson J, Berry P, Ebi K, et al. Health care facilities resilient to climate change impacts. Int $J$ Environ Res Public Health 2014;11:13097-116.

25 Poland B, Dooris M. A green and healthy future: the settings approach to building health, equity and sustainability. Crit Public Health 2010;20:281-98.

26 Hess JJ, McDowell JZ, Luber G. Integrating climate change adaptation into public health practice: using adaptive management to increase adaptive capacity and build resilience. Environ Health Perspect 2012;120:171-9.

27 Arksey H, O'Malley L. Scoping studies: towards a methodological framework. Int J Soc Res Methodol 2005;8:19-32.

28 Levac D, Colquhoun H, O'Brien KK. Scoping studies: advancing the methodology. Implementation Sci 2010;5.

29 Tricco AC, Lillie E, Zarin W, et al. PRISMA extension for scoping reviews (PRISMA-ScR): checklist and explanation. Ann Intern Med 2018;169:467. 